\title{
Effect of daily oral minodronate on vertebral fractures in Japanese postmenopausal women with established osteoporosis: a randomized placebo-controlled double-blind study
}

\author{
T. Matsumoto • H. Hagino • M. Shiraki • M. Fukunaga • \\ T. Nakano • K. Takaoka • H. Morii • Y. Ohashi • \\ T. Nakamura
}

Received: 19 March 2008 / Accepted: 17 October 2008 / Published online: 20 December 2008

(C) The Author(s) 2008. This article is published with open access at Springerlink.com

\begin{abstract}
Summary A randomized placebo-controlled trial was conducted to examine the effect of daily oral $1 \mathrm{mg}$ minodronate on vertebral fractures in 704 postmenopausal women with established osteoporosis for 24 months. Minodronate treatment reduced vertebral fractures by $59 \%$ without serious adverse events. Minodronate is a safe and effective bisphosphonate for osteoporosis treatment.

Introduction Minodronate increases bone mineral density (BMD) in postmenopausal osteoporotic patients. However, its efficacy in reducing osteoporotic fractures has not been tested.
\end{abstract}

ClinicalTrials.gov Identifier: NCT00212667.

T. Matsumoto $(\bowtie)$

Department of Medicine and Bioregulatory Sciences,

University of Tokushima Graduate School of Medical Science,

3-18-15 Kuramoto-cho,

Tokushima 770-8503, Japan

e-mail: toshimat@clin.med.tokushima-u.ac.jp

H. Hagino

Rehabilitation Division, Tottori University Hospital,

Yonago, Japan

\section{Shiraki}

Research Institute and Practice for Involutional Diseases,

Nagano, Japan

M. Fukunaga

Department of Nuclear Medicine, Kawasaki Medical School,

Okayama, Japan

\section{T. Nakano}

Department of Orthopaedic Surgery, Tamana Central Hospital,

Kumamoto, Japan
Methods To examine anti-fracture efficacy and safety of daily oral minodronate in postmenopausal women with established osteoporosis, a randomized, double-blind, placebo-controlled trial was conducted in 704 postmenopausal women (55 to 80 years) with one to five vertebral fractures and low BMD. Subjects were randomly assigned to receive daily oral $1 \mathrm{mg}$ minodronate $(n=359)$ or placebo $(n=345)$ for 24 months, with daily supplements of $600 \mathrm{mg}$ calcium and $200 \mathrm{IU}$ vitamin $\mathrm{D}_{3}$.

Results Daily $1 \mathrm{mg}$ minodronate for 24 months reduced the risk of vertebral fractures by 59\% (95\% CI, 36.6-73.3\%). Furthermore, when fractures during the first 6 months were
K. Takaoka

Department of Orthopedic Surgery,

Osaka City University Graduate School of Medicine, Osaka, Japan

\section{H. Morii}

Osaka City University Medical School,

Osaka, Japan

Y. Ohashi

Department of Biostatistics, School of Public Health, University of Tokyo,

Tokyo, Japan

T. Nakamura

Department of Orthopedic Surgery,

University of Occupational and Environmental Health,

Fukuoka, Japan 
eliminated, the risk of vertebral fractures from 6 to 24 months was reduced by $74 \%$ in minodronate-treated group. Minodronate treatment also reduced height loss. Bone turnover markers were suppressed by about $50 \%$ after 6 months of minodronate treatment and remained suppressed thereafter. The overall safety profile including gastrointestinal safety was similar between the two groups. Conclusions Daily oral minodronate is safe, well-tolerated, and is effective in reducing vertebral fracture risk in postmenopausal women with established osteoporosis.

Keywords Bisphosphonate - Bone turnover markers . Fracture prevention $\cdot$ Height loss $\cdot$ Minodronate

\section{Introduction}

More than one fourth of women in their 70s suffer from at least one osteoporotic vertebral fracture $[1,2]$. Incidence of new fractures rises with increasing number of preexisting fractures [3], and not only morbidity but also mortality rate rises with increasing number of fractures [4, 5]. Thus, osteoporosis has become a significant socioeconomic burden in aged societies.

Bisphosphonates have been shown to have potent antifracture efficacy by inhibiting bone resorption, with a reduction in bone turnover and an increase in bone mineral density (BMD). Minodronate (ONO-5920/YM529) is a nitrogen-containing bisphosphonate with potent inhibitory effect on bone resorption [6]. Previous in vitro and in vivo preclinical studies demonstrated that minodronate is about ten times as potent as alendronate in inhibiting bone resorption [7]. A randomized placebo-controlled doubleblind trial revealed that daily oral administration of $0.5,1.0$, and $1.5 \mathrm{mg}$ minodronate to Japanese women with postmenopausal osteoporosis for 9 months caused an increase in lumbar BMD by $4.9 \%, 5.7 \%$, and $5.2 \%$, respectively, compared with the placebo group [8]. Because the incidence of adverse gastrointestinal events did not increase in a dose-dependent manner $(0 \%, 12.6 \%, 6.3 \%$, and $11.1 \%$ by placebo, $0.5,1.0$, and $1.5 \mathrm{mg}$ minodronate treatment, respectively), minodronate was shown to be well tolerated with excellent effect in increasing BMD. In addition, a head-to-head comparison of the effects of daily oral $1 \mathrm{mg}$ minodronate with $5 \mathrm{mg}$ alendronate revealed that the effect of 12-month treatment with $1 \mathrm{mg}$ minodronate on lumbar and total hip BMD was similar to those of $5 \mathrm{mg}$ alendronate and that minodronate was generally well tolerated with similar safety profiles to alendronate (Hagino et al., submitted for publication). These data suggest that minodronate can become a new treatment choice as a potent bisphosphonate for patients with established osteoporosis. However, its efficacy in reducing osteoporotic fractures has not been evaluated. The present phase III clinical trial was conducted to examine the effect of daily oral $1 \mathrm{mg}$ minodronate on the prevention of vertebral fractures in Japanese women with postmenopausal osteoporosis.

\section{Materials and methods}

Patient enrollment

We studied postmenopausal women aged 55 to 80 with one to five fragility fractures between the vertebrae $\mathrm{T} 4$ and L4 and BMD below $80 \%$ ( $T$ score -1.7 at the lumbar spine) of the young adult mean (YAM) [9]. Data for the YAM and $T$ score values were obtained from the reference data in 3,218 Japanese healthy women with 20 to 44 years of age [10].

Subjects were excluded if they had disorders such as primary hyperparathyroidism, Cushing's syndrome, premature menopause due to hypothalamic, pituitary or gonadal insufficiency, poorly controlled diabetes mellitus ( $\mathrm{HbAlc}$ over $8.0 \%$ ), or other causes of secondary osteoporosis, or if they had any radiographic finding that might affect the assessment of vertebral fractures and used hard or semihard corset in spine part. Subjects with peptic ulcer were excluded. Subjects were excluded if they had taken bisphosphonates at any time. Subjects were also excluded if they had taken glucocorticoids, calcitonin, vitamin K, active vitamin D compounds, or hormone replacement therapy within the previous 2 months, had serum calcium (Ca) levels above $10.6 \mathrm{mg} / \mathrm{dL}(2.7 \mathrm{mmol} / \mathrm{L})$ or below $8.0 \mathrm{mg} / \mathrm{dl}(2.0 \mathrm{mmol} / \mathrm{L})$, had serum creatinine levels above $1.5 \mathrm{mg} / \mathrm{dL}(133 \mu \mathrm{mol} / \mathrm{L})$, or had clinically significant hepatic disorders.

This study was conducted in accordance with consideration for the protection of patients, as outlined in the Declaration of Helsinki, and was approved by the appropriate institutional review boards. All subjects gave written informed consent before undergoing any examination or study procedure, which was conducted in compliance with Good Clinical Practice.

\section{Study design}

This study was a randomized, double-blind, placebocontrolled, multicenter study at 98 sites in Japan. Subjects who met all the entry criteria were enrolled and sequentially assigned an allocation number independent of study site. Subjects were randomized to take $1 \mathrm{mg}$ minodronate (Astellas Pharma, Tokyo, Japan) or placebo once a day and were treated for 24 months. Randomization was performed by a computerized system. Subjects were instructed to take their tablet on rising and $30 \mathrm{~min}$ before food with plain water. All 
subjects received daily calcium $(600 \mathrm{mg})$ and vitamin D (200 IU) supplementation once a day after the evening meal. Adherence with the study treatment was assessed with the use of medication diaries and counts of residual medication supplies.

\section{Study outcomes}

The primary endpoint of the study was the cumulative proportion of patients with new morphometric vertebral fractures at 24 months of treatment with the study medication. Secondary endpoints included length of the period to the occurrence of new vertebral fractures, the risk of patients and length of the period to the occurrence of clinical fractures, changes in height, and relative changes in bone turnover markers.

Assessment of vertebral fractures Lateral radiographs of the thoracic and lumbar spine were taken at the screening visit to determine the presence of prevalent fractures. Subjects were enrolled based on a visual assessment of prevalent fractures in T4 to L4. All the radiologic specifications and the levels of vertebra at the thoracic sand lumbar spine were standardized throughout the study sites. The assessment of prevalent fractures was made if the ratio of anterior or middle vertebral body height to the posterior vertebral body height was less than 0.8 [11]. Quantitative and semiquantitative techniques $[12,13]$ were used to identify incident vertebral fractures for the purposes of the efficacy determination. Lateral radiographs of the spine were performed at $6,12,18$, and 24 months for the assessment of incident fractures. An incident of new vertebral fracture was diagnosed if the anterior, posterior, or middle vertebral height had decreased by at least $15 \%$ and by $4 \mathrm{~mm}$ in a vertebra that was normal at baseline, or semiquantitatively as a progress in grades [11]. Morphological diagnosis of fractures was made by quantitative and semiquantitative assessment of two evaluators who were blinded to the sequence of films at two independent central reading facilities at Tottori University, Yonago, Japan by Hagino, $\mathrm{H}$. and at the University of Occupational and Environmental Health, Fukuoka, Japan by Nakamura, T., with adjudication by a third investigator (Nakano,T. at Tamana Central Hospital, Kumamoto, Japan) in the event of discrepant results.

Assessment of non-vertebral fractures All non-vertebral fractures were identified symptomatically as clinical fractures, and only non-traumatic fractures assessed by investigators were reported. Suspected clinical fractures at six non-vertebral sites (humerus, radius/ulna, subclavia, pelvis, femur, and tibia/fibula) were adjudicated radiographically, and only radiographically confirmed fractures were listed.
Assessment of bone turnover Serum and urine samples were collected at baseline, $6,12,18$, and 24 months for measurement of bone turnover markers, including urinary total deoxypyridinoline (DPD) measured by high-performance liquid chromatography (SRL, Tokyo, Japan) [14] after acid hydrolysis, urinary type I collagen $N$-telopeptide (NTX; Osteomark, Ostex International, Seattle, WA, USA), serum bone-specific alkaline phosphatase (BALP; Osteolinks "BAP", Quidel, San Diego, CA, USA), serum osteocalcin (BGP-IRMA Mitsubishi; Mitsubishi Kagaku Iatron, Tokyo, Japan), and serum 25-hydroxyvitamin D (25 (OH)D; 125I RIA Kit, DiaSorin Inc., Saluggia, Italy). Study subjects were asked to visit study sites during the morning, but it was not mandatory to visit in the fasting state.

Assessment of adverse events All subjects were questioned about adverse events (AEs) of treatment at each visit, and all adverse events reported were analyzed regardless of the investigators' assessments of causality. The Medical Dictionary for Regulatory Activities (MedDRA, Version 8.1J) was used to categorize reported adverse events.

Statistical analysis All the data analyses were performed by statisticians from Ono under the supervision and confirmation of data analyses by one of the authors (Ohashi, Y.).

The intention-to-treat (ITT) population comprised all patients who received at least one dose of study medication and who attended at least one follow-up visit for any observation of efficacies. The ITT population was used for all fracture and height analyses. Safety analyses population comprised all patients who received at least one dose of study medication in either treatment group. A per-protocol (PP) approach was used as a primary approach to analyze the bone turnover markers because they can change rapidly by protocol violations, interruption of study therapy, or concurrent illness. The PP approach excluded protocol violators who took less than $75 \%$ study drug, who took prohibited medications during the course of the trial, or who violated the protocol in a significant manner as specified in the data analysis plan, and patients who took study drug for less than 12 months. This population included all patients in the ITT population, except those with a protocol deviation deemed to have a significant impact on the efficacy variables, i.e., major deviations regarding the inclusion/exclusion criteria, patients with insufficient compliance $(<75 \%$ of the study medication), documentation of forbidden concomitant medication that could bias the fracture results, and patients lacking an assessable baseline and follow-up for X-ray assessments for less than 12 months.

The risk of patients with new morphometric vertebral fractures at 24 months, as the primary endpoint, was 
analyzed by testing the superiority of minodronate group to the placebo group by the time-to-event curves (Kaplan-Meier method), the event being the first new incident vertebral fracture. The primary hypothesis was tested using an ITT analysis that was modified to include all subjects randomized, who had taken at least one dose of study drug, and attended at least one follow-up visit. A Cox regression model was used to estimate the relative risk of vertebral fracture and its 95\% confidence interval in minodronate group and placebo group. Log-rank test was used to determine the superiority of the minodronate group to the placebo group. The power calculation was based on the predictive risk of vertebral fracture. For the study to achieve a power of $90 \%$ to detect the superiority, a sample size of 290 subjects per group was required. To allow for subject withdrawals, the intention was to enroll 640 to 650 subjects. Differences were considered to be statistically significant if the $p$ value was less than 0.05 .

Group mean and standard error (SE) were given for the percent changes from baseline in bone turnover markers and changes from baseline in height and were used to assess the significance of changes within two groups. $T$ test was used to determine whether minodronate group was significantly different from the placebo group. The comparability between minodronate and placebo groups for demographic information was assessed with Wilcoxon's rank-sum test or Fisher's exact test. Differences in proportions of patients with AEs were analyzed using Fisher's exact test. The treatment groups were also compared for the proportion of patients with gastrointestinal AEs using Fisher's exact test. Statistical analyses were performed using Statistical Analysis Systems (SAS Institute, Cary, NC, USA). All protocol violators were identified before database lock of the study.

\section{Results}

\section{Patient disposition}

A total of 1,083 subjects were screened at 98 study sites in Japan (Fig. 1). A total of 704 subjects were randomized to take either minodronate (359 subjects) or placebo (345 subjects). Five patients in the minodronate group and three patients in the placebo group were excluded from the safety analysis population for reasons of not receiving the study medication or withdrawal of informed consent. Among the safety analysis population, a total of 161 had been treated with either $20 \mathrm{IU} /$ week calcitonin (154 subjects) or estrogen (seven subjects) before the washout period. None of the study subjects were given glucocorticoid treatment before enrollment. The proportion of the subjects in the ITT analysis $(95.5 \%$ and $95.9 \%$ in minodronate and placebo groups, respectively) and PP analysis (75.5 and $76.2 \%$ in minodronate and placebo groups, respectively) was similar between the two groups.

\section{Baseline characteristics of the subjects}

The baseline demographics of subjects were well balanced between the two groups (Table 1). The number of vertebral fractures at baseline was not significantly different, and the number of subjects with one, two, and three or more vertebral fractures was similar between the two groups. There was no significant difference in lumbar BMD, serum $25(\mathrm{OH}) \mathrm{D}$, and the levels of bone turnover markers at the baseline between the two groups.

\section{Vertebral fractures}

After 24 months of treatment, there was a statistically significant reduction in the risk of vertebral fractures in the minodronate group compared with the placebo group $(p<0.0001$, log-rank test; Fig. 2). The Kaplan-Meier estimates of risk after 24 months of treatment were $10.4 \%$ in the minodronate group and $24.0 \%$ in the placebo group of the ITT population. Relative risk of vertebral fractures by minodronate treatment was 0.411 (95\% confidence interval [CI], 0.267-0.634), and relative risk reduction rate in cumulative fracture incidence by minodronate treatment was $59 \%$. Among patients in the PP population who completed the 2-year study ( $n=253$ in the minodronate group and $n=239$ in then placebo group), the incidence of vertebral fractures was $9.9 \%$ in the minodronate group and $21.3 \%$ in the placebo group. These numbers were very similar to those observed in the ITT population.

A large number of fractures occurred during the first 6 months in both groups (20 and 27 in minodronate and placebo groups, respectively), and the decrease in vertebral fracture risk by minodronate treatment was more pronounced after the initial 6 months until the end of the study period (Table 2). When the incidence of vertebral fractures during the first 6 months was compared between subgroups with one prevalent fracture and two or more fractures, the incidence of vertebral fractures during the first 6 months was five $(3.5 \%)$ in minodronate group and six $(4.3 \%)$ in placebo group among patients with one prevalent fracture. In contrast, vertebral fracture incidence during the first 6 months was $15(9.0 \%)$ in the minodronate group and 21 $(12.3 \%)$ in the placebo group among patients with two or more prevalent fractures. Thus, majority of the fractures during the early study period came from patients with two or more prevalent fractures. 
Fig. 1 Enrollment and outcomes. A total of 1,083 subjects were screened, and 704 subjects were randomized to take either minodronate (359 subjects) or placebo (345 subjects)

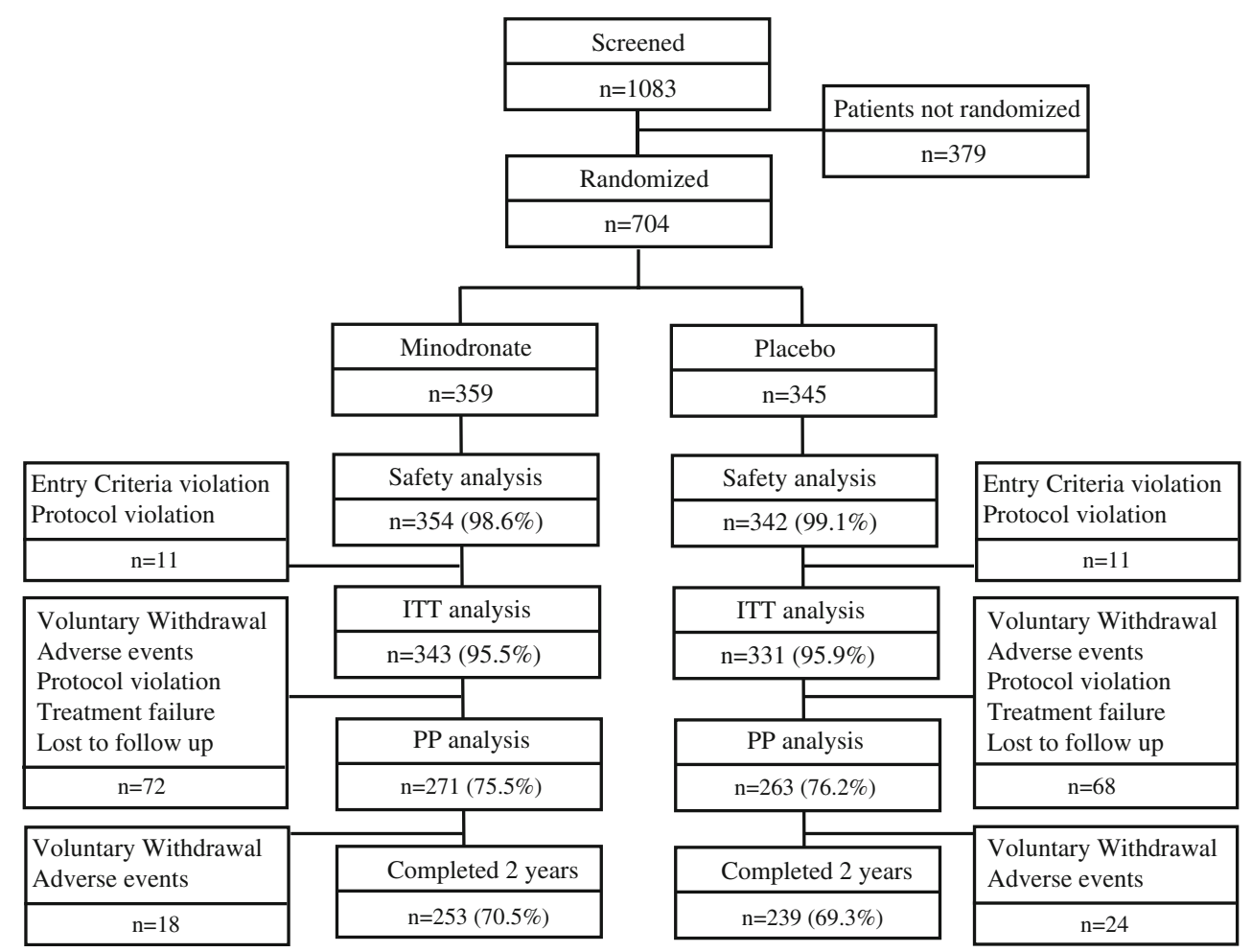

\section{Height loss}

In order to examine whether the effect of minodronate on vertebral fracture prevention was related to the stature of patients, height of studied subjects was assessed at 12 and 24 months using stature meter equipped at each study site. At 12 months, a mean stature loss in the minodronate group (1.2 $\mathrm{mm})$ was already significantly less than that in the placebo group (3.4 mm; $p<0.05$ ) (Fig. 3a). After 24 months of treatment, a mean stature loss of $6.8 \mathrm{~mm}$ was observed in the placebo group, which was significantly larger than that in the minodronate group (3.7 mm, $p<0.01$; Fig. 3a). There was no significant height loss in those patients without fracture, and in those patients who did not fracture, no significant effect of minodronate treatment on the height was observed (Fig. 3b).

\section{Non-vertebral fractures}

Non-vertebral fractures that occurred during the trial were picked up from the report of clinical fractures and confirmed by radiographs. Because the number of subjects in each group was small and the study period was short, no significant difference was observed between the groups

Table 1 Demographics and baseline characteristics of subjects

\begin{tabular}{lll}
\hline Characteristic & Minodronate $(n=343)$ & Placebo $(n=331)$ \\
\hline Age $($ years $)$ & $71.4[6.0]$ & $71.7[5.6]$ \\
Height $(\mathrm{cm})$ & $147.6[5.9]$ & $147.0[5.9]$ \\
Body mass index $\left(\mathrm{kg} / \mathrm{m}^{2}\right)$ & $23.4[3.1]$ & $23.5[3.3]$ \\
Time since menopause $($ years $)$ & $21.3[7.2]$ & $22.2[6.8]$ \\
Number of prevalent vertebral fractures & $2.0[1.2]$ & $2.1[1.2]$ \\
With one fracture $[n(\%)]$ & $161(46.9)$ & $147(44.4)$ \\
With two fractures $[n(\%)]$ & $88(25.7)$ & $80(24.2)$ \\
With three or more fractures $[n(\%)]$ & $94(27.4)$ & $104(31.4)$ \\
Lumbar BMD $T$ score & $-2.95[0.77]$ & $-2.95[0.77]$ \\
Serum $25(\mathrm{OH}) \mathrm{D}(\mathrm{ng} / \mathrm{mL})$ & $25.0[6.0]$ & $25.4[6.2]$ \\
Serum BALP $(\mathrm{U} / \mathrm{L})$ & $33.0[11.8]$ & $33.4[13.0]$ \\
Serum osteocalcin $(\mathrm{ng} / \mathrm{mL})$ & $9.1[2.8]$ & $9.2[3.1]$ \\
Urine total DPD $(\mathrm{pmol} / \mu \mathrm{mol} \mathrm{Cr})$ & $8.8[3.6]$ & $8.9[3.1]$ \\
Urine NTX $(\mathrm{nmol} \mathrm{BCE} / \mathrm{mmol} \mathrm{Cr})$ & $50.2[24.0]$ & $50.9[21.9]$ \\
\hline
\end{tabular}

Data are means [SD] for the indicated number of subjects in each group. 


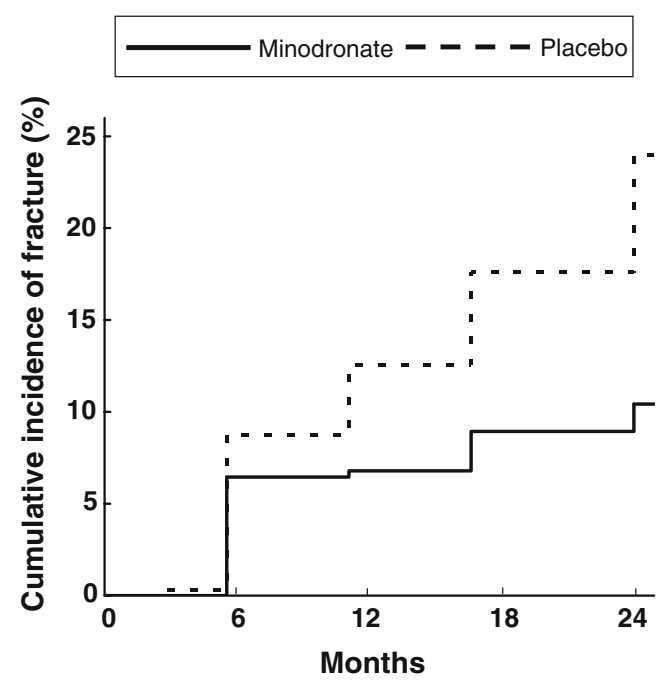

Fig. 2 Kaplan-Meier estimates of the effect of daily oral $1 \mathrm{mg}$ minodronate for 24 months on the risk of vertebral fractures in osteoporotic subjects. Cumulative incidence of vertebral fractures from the start of the study. Minodronate treatment reduced relative risk of vertebral fractures by $59 \%$

with daily $1 \mathrm{mg}$ minodronate and placebo in the incidence of non-vertebral fractures at the major six sites (radius/ulna, humerus, femur, tibia/fibula, subclavia, and pelvis) after 24 months of treatment $(2.7 \%$ in the minodronate and $3.5 \%$ in the placebo group).

Bone turnover markers

Bone turnover markers decreased significantly in the minodronate group, compared with in the placebo group $(p<0.0001)$. Mean percent changes in bone resorption markers, urinary DPD and NTX, at 6 months were $-42.4 \%$ and $-49.5 \%$, respectively, in the minodronate group, compared with $-4.0 \%$ and $-7.9 \%$, respectively, in the placebo group. Bone resorption markers remained almost constant thereafter until 24 months of treatment, when the reduction in urinary DPD and NTX in the minodronate group was $-37.1 \%$ and $-56.7 \%$, respectively (Fig. 4a, b). Bone formation markers, BALP and osteocal- cin, also decreased at 6 months by $-46.2 \%$ and $-45.5 \%$, respectively, in the minodronate group, compared with $-14.1 \%$ and $-16.3 \%$, respectively, in the placebo group. Bone formation markers also remained almost constant until 24 months of treatment, and reduction in BALP and osteocalcin from baseline was $-51.7 \%$ and $-50.9 \%$ in the minodronate group, respectively (Fig. 4c, d).

\section{Adverse events}

The overall incidence of AEs was similar in both groups, as was the incidence of gastrointestinal AEs, drug-related AEs, and serious AEs (Table 3). The most common gastrointestinal tract AEs were constipation, gastric discomfort, and diarrhea. Among serious AEs, more patients in minodronate group reported infections/infestations and cardiac disorders. Infections included two pneumonia patients in both minodronate and placebo groups, and all the other infections were reported in only one patient in either group. Cardiac disorders included three patients in minodronate and two patients in placebo group with ischemic heart diseases, and one patient each with cardiac insufficiency and sinus arrhythmia in minodronate group. None of them reported atrial fibrillation. The proportion of subjects who discontinued the study due to AEs was also similar between the two groups. Complaints related to digestive system were the most common AEs associated with withdrawal from the study (Table 3).

\section{Discussion}

The present study demonstrated that daily oral administration of $1 \mathrm{mg}$ minodronate for 24 months reduced the risk of new vertebral fractures by $59 \%$ compared with that in the placebo group. The effect of minodronate on vertebral fracture was observed within 12 months, and there was also a significant decrease in height loss at 12 months. The overall safety profile including gastrointestinal safety was similar between the two groups.

Table 2 Cumulative incidence of vertebral fractures

\begin{tabular}{|c|c|c|c|c|c|c|c|}
\hline \multirow[t]{2}{*}{ Months } & \multicolumn{3}{|c|}{ Minodronate } & \multicolumn{3}{|c|}{ Placebo } & \multirow{2}{*}{$\begin{array}{l}\text { Log-rank } \\
\text { test }\end{array}$} \\
\hline & $n$ & $\begin{array}{l}\text { Number of } \\
\text { patients }(\%)\end{array}$ & $\begin{array}{l}\text { Cumulative } \\
\text { incidence (\%) }\end{array}$ & $n$ & $\begin{array}{l}\text { Number of } \\
\text { patients }(\%)\end{array}$ & $\begin{array}{l}\text { Cumulative } \\
\text { incidence (\%) }\end{array}$ & \\
\hline 0 & 339 & $0(0.0)$ & 0.0 & 328 & $0(0.0)$ & 0.0 & $P<0.0001$ \\
\hline 6 & 310 & $20(6.5)$ & 6.5 & 308 & $27(8.7)$ & 8.7 & \\
\hline 12 & 274 & $1(0.4)$ & 6.8 & 265 & $11(4.2)$ & 12.5 & \\
\hline 18 & 261 & $6(2.3)$ & 8.9 & 242 & $14(5.8)$ & 17.6 & \\
\hline 24 & 246 & $4(1.6)$ & 10.4 & 219 & $17(7.8)$ & 24.0 & \\
\hline
\end{tabular}

Data was analyzed by actuarial method. 

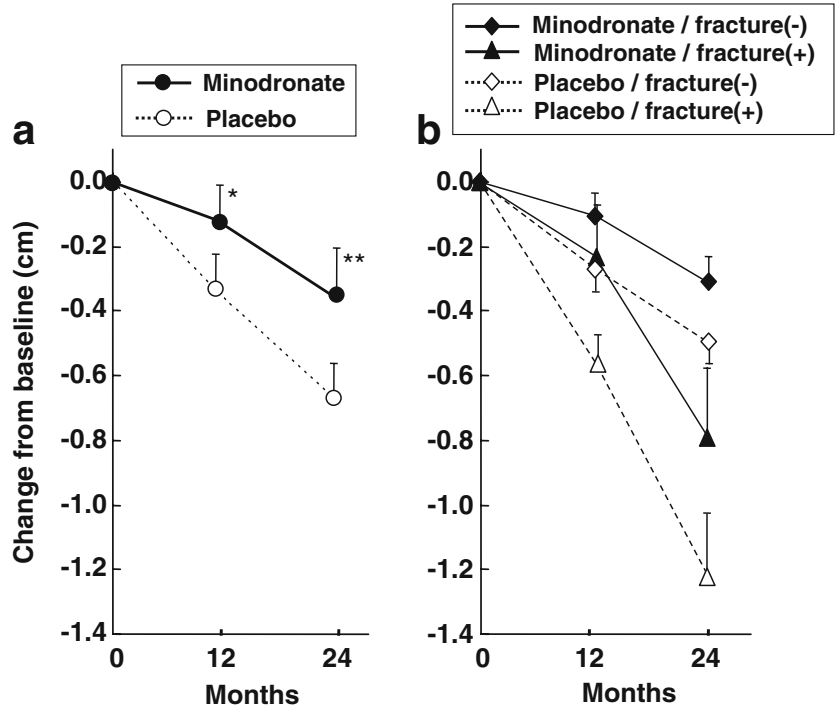

Fig. 3 Effect of daily oral $1 \mathrm{mg}$ minodronate for 24 months on height changes of osteoporotic patients. a Minodronate treatment significantly reduced height reduction at both 12 months $\left({ }^{*} p<0.05\right)$ and 24 months $(* * p<0.01)$. b Height changes in minodronate-treated patients with (closed triangle, $n=27$ ) or without (closed diamond, $n=242$ ) vertebral fracture, and placebo-treated patients with (open triangle, $n=61$ ) or without vertebral fracture (open diamond, $n=200$ ) are shown. Data are means \pm SE

In the present study, a large number of vertebral fractures occurred during the first 6 months in both groups (20 and 27 in minodronate and placebo groups, respectively). In our previous study, to compare the effect of minodronate on lumbar BMD and bone markers with that of alendronate (Hagino et al., submitted for publication), bone resorption markers were suppressed within 1 month, and lumbar BMD was significantly increased after 3 months of minodronate treatment. It should be noted that the assessment of vertebral fractures at baseline was performed within 2 months before the start of study drug administration. Therefore, a part of vertebral fractures identified after 6 months of drug administration might have occurred before drug administration was started. Although the exact reason why a large number of vertebral fractures occurred during the early period in both groups remains unclear, minodronate showed a marked anti-fracture efficacy from 6 to 24 months of treatment (Table 2).

In contrast to the robust inhibitory effect on vertebral fractures, the present study did not show a significant effect of minodronate in reducing non-vertebral fractures. This is a major limitation of the present study. Because the study was aimed to examine the ability of minodronate to reduce the risk of vertebral fractures, the study did not have enough power in terms of the number of study subjects and the length of study period to examine the effect of minodronate on non-vertebral fractures. Thus, although the study included patients with established osteoporosis having at least one prevalent vertebral fracture, the number of non-vertebral fractures developed in long bones during the 24-month study period was too small to draw any conclusions.

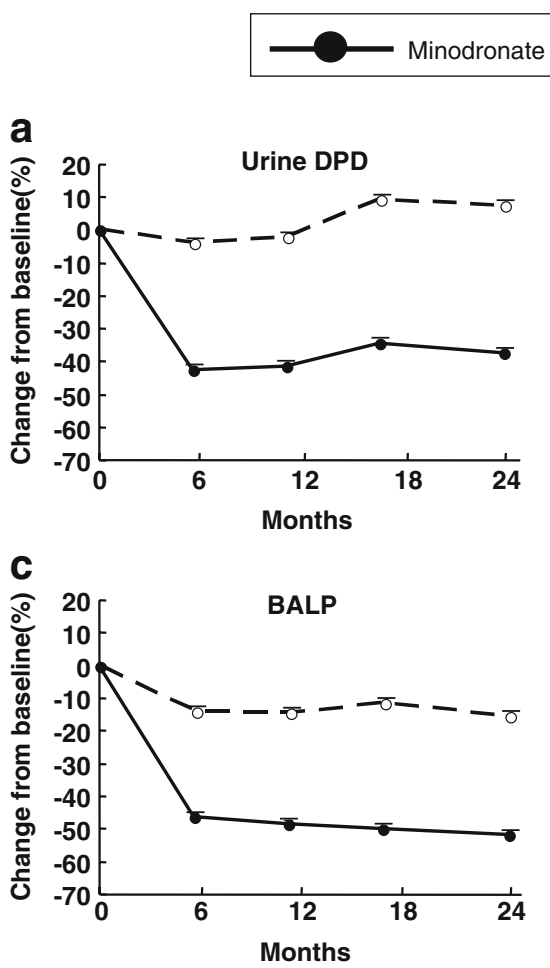

- - - Placebo
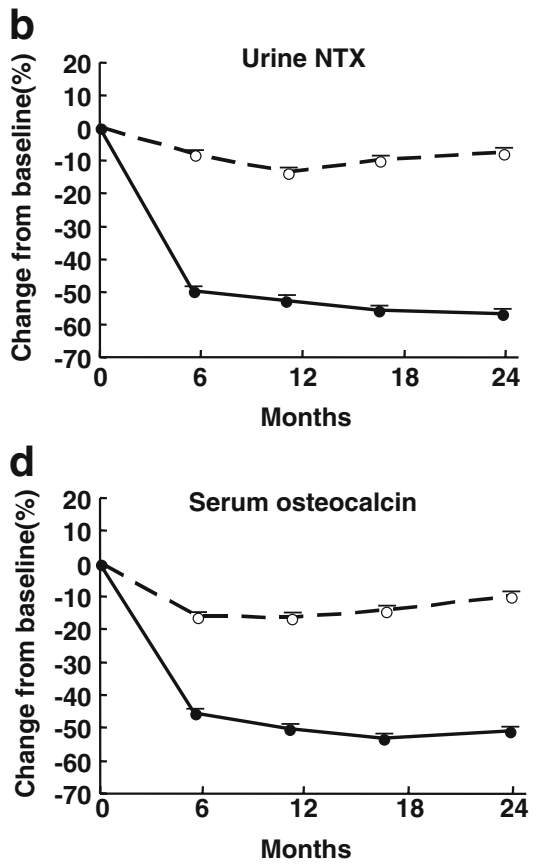

Fig. 4 Effect of daily oral $1 \mathrm{mg}$ minodronate for 24 months on the changes in bone turnover markers in osteoporotic patients. Data are means \pm SE 
Table 3 Summary of adverse events

\begin{tabular}{lll}
\hline & Minodoronate, $n(\%)$ & Placebo, $n(\%)$ \\
\hline No. of patients & 354 & 342 \\
Any AE & $334(94.4)$ & $327(95.6)$ \\
Gastrointestinal AE & $173(48.9)$ & $155(45.3)$ \\
"Drug-related" AE & $54(15.8)$ \\
Serious AE & $57(16.1)$ & $65(19.0)$ \\
Injury, poisoning and procedural complications & $49(13.8)$ & $13(3.8)$ \\
Musculoskeletal and connective tissue disorders & $10(2.8)$ & $9(2.6)$ \\
Gastrointestinal disorders & $8(2.3)$ & $9(2.6)$ \\
Nervous system disorders & $7(2.0)$ & $10(2.9)$ \\
Infections and infestations & $4(1.1)$ & $3(0.9)$ \\
Eye disorders & $7(2.0)$ & $8(2.3)$ \\
Respiratory, thoracic and mediastinal disorders & $1(0.3)$ & $5(1.5)$ \\
Cardiac disorders & $3(0.8)$ & $2(0.6)$ \\
Neoplasms benign, malignant and unspecified & $5(1.4)$ & $4(1.2)$ \\
Discontinued due to AE & $2(0.6)$ & $47(13.7)$ \\
Discontinued due to gastrointestinal AE & $55(15.5)$ & $13(3.8)$ \\
Discontinued due to "drug-related" AE & $17(4.8)$ & $14(4.1)$ \\
\hline
\end{tabular}

Data are number of patients

$A E$ adverse event

a AEs reported as drug-related by the investigators are listed as "drug-related"

${ }^{\mathrm{b}}$ Serious AEs with more than two patients in either treatment group are listed

With regard to the safety profile of minodronate, no significant difference was observed between the minodronate and placebo groups in any AEs including drug-related or serious AEs. Although the most common AEs were gastrointestinal AEs, the incidence of gastrointestinal AEs, as well as those that caused discontinuation from the study, was very similar between the minodronate and placebo groups. These results suggest that minodronate does not cause any serious disturbance in osteoporotic patients, and daily administration of minodronate can be well-tolerated in patients with osteoporosis.

Minodronate exhibits very similar antiresorptive potency to zoledronic acid in pre-clinical studies [7], and intermittent oral administration of ibandronate [15] as well as yearly intravenous administration of zoledronic acid [16] demonstrated potent anti-fracture efficacy. Therefore, further studies are warranted to examine the effect of intermittent oral and intravenous minodronate on vertebral and non-vertebral fractures in osteoporotic patients.

In conclusion, daily oral minodronate is safe, welltolerated, and is effective in reducing vertebral fracture risk in postmenopausal women with established osteoporosis. Because the dose of minodronate in reducing fracture incidence was low, further studies are warranted to evaluate the efficacy of intermittent administration of higher doses of minodronate on osteporotic fractures.

Acknowledgments We thank Mr. T. Minamide and Mr. T. Matsuoka, ONO Pharmaceutical Co., Ltd., for their scientific and technical support, Astellas Pharmaceutical, for providing supportive data, and the following investigators and clinical sites in Japan that participated in this study: T. Ota, Ota Orthopedic Clinic; M. Kokaji, M. Tsuji, S. Kawamura, Kobayashi Hospital; T. Hashimoto, Hakodate Koseiin Hakodate Central General Hospital; S. Sato, Eniwa Hospital; G. Katahira, Sapporo Kiyota Orthopeadic Hospital; Y. Saito, Hokuei Orthopedics; S. Nabeshima, Nabeshima Clinic; T. Fukunaga, Ainosato Orthopedics; T. Chiba, Kikusui Orthopedics; H. Yamamoto, Toyohira Orthopedics; H. Koga, Koga Orthopedic Clinic; T. Ando, Morioka Hospital; S. Tsukikawa, Tsukikawa Lady's Clinic; S. Harada, Tsukuba Gakuen Hospital; N. Tajima, Tajima Geka Ichouka; K. Ogata, Seiwakai Shoda Hospital; T. Michimata, Uchibori Seikeigeka Iin; H. Inoue, Inoue Hospital; M. Inuzuka, Chousei Hospital; S. Ichikawa, Cardiovascular Hospital of Center Japan; K. Toba, Toba Orthopedic Clinic; H. Sato, Saiseikai Kawaguchi General Hospital; Y. Kaneda, Kaneda Orthopedics; K. Inoue, Tokyo Women`s Medical University Medical Center East; S. Yamada, Kyoai Clinic; K. Fukuda, Shiratori Clinic; S. Sano, Sanraku Hospital; A. Yamaguchi, Yamaguchi Hospital; T. Nakamura, Abe Clinic; K. Maruyama, Gate Town Hospital; T. Nakagawa, Senpo Tokyo Takanawa Hospital; T. Takemoto, Misyuku Hospital; K. Kamada, Kumegawa Hospital; H. Mizuguchi, T. Ryu, Y. Sakamoto, S. Katayama, Mizuguchi Hospital; R. Kimura, Hideshima Hospital; S. Yamaguchi, Gonohashi Clinic; C. Nokubo, Nokubo Orthopedic Clinic; M. Takemoto, Takemoto Orthopedics; T. Ishihara, Shirahigebashi Hospital; Y. Tsuruta, Tsuruta Clinic; S. Yamazaki, Sengoku Hospital; T. Ishibashi, T. Okubo, Oguchi East Hospital; K. Suzuki, A. Okazaki, Shonan Daiichi Hospital; H. Machida, Kanto Rosai Hospital; S. Yamashita, Hayama Orthopedics; Y. Mikami, Yokohama Rosai Hospital; I. Miyata, Aoba Orthopedics Clinic; M. Kasuga, Kasuga Orthopedics; M. Tsuboi, Yokohama Minoru Clinic; N. Nagata, Nagata Orthopedics; N. Endo, Niigata University Medical \& Dental Hospital; Y. Murai, Murai Orthopedic Iin; S. Noto, Noto Orthopedics; M. Katsumi, Katsumi Orthopedics; H. Morishita, T. Takino, Kanazawa Social Insurance Hospital; N. Hachisuka, Hachisuka Orthopedics; M. Takimori, Nirasaki Mutual Hospital; Y. Nagasaka, Nagasaka Orthopedics; M. Suzuki, Suzuki Orthopedic Iin; S. Kumaki, Hokushin General Hospital; S. Kobayashi, Shinsyu University Hospital; T. Hanaoka, Yamabe Spa Hanaoka 
Orthopedics; H. Misawa, Yodakubo Hospital; M. Shiraki, Research Institute and Practice for Involutional Diseases; S. Tsuboi, Shizuoka Kosei Hospital; K. Yamazaki, Hamamatsu University School of Medicine University Hospital; M. Taniguchi, Taniguchi Orthopedic Iin; M. Fukuchi, Aobadai Fukuchi Orthopedics \& Gastroenterology Clinic; M. Denda, Denda Orthopedics; Y. Nishijima, Nishijima Hospital; T. Kitakoji, Nagoya University Hospital; Y. Hachiya, Hachiya Orthopedic Hospital; Y. Osaka, Minamiosaka Hospital; A. Tei, Kishiwada Tokushukai Hospital; Y. Honda, Baba Memorial Hospital; N. Sha, Kanebo Memorial Hospital; T. Noda, C. Terada, Ako Central Hospital; J. Sako, Irie Hospital; Y. Higashi, Himeji Central Hospital; T. Onishi, Onishi Orthopedics; T. Matsubara, Matsubara Clinic; Y. Koyama, Matsubara Mayflower Hospital; S. Soen, Kinki University School of Medicine, Nara Hospital; M. Ozaki, Kitade Hospital; M. Ohama, Yonago East Hospital; T. Nishiyama, Tamashima Daiichi Hospital; H. Sanada, Sanada Hospital; K. Sanuki, Sanuki Orth \& Rheumatic Clinic; T. Taguchi, Yamaguchi University Hospital; S. Yamagata, Yamagata Iin; K. Nobutani, Sea Side Hospital; H. Yamazaki, H. Ueno, Mine City Hospital; S. Ono, Marugame Ono Clinic; A. Kuge, S. Morita, Izumino Hospital; T. Ogata, Ogata Orthopedic Hospital; H. Ikematsu, Haradoi Hospital; A. Iwaki, K. Domen, Okabe Hospital; Y. Ishibashi, Ishibashi Orthopedics; T. Tsuruta, Tsuruta Orthopaedic Clinic; H. Shibata, Shibata Chokodo Hospital; T. Segata, Kumamoto Saishunso National Hospital; T. Naono, Oita Oka Hospital; E. Nakamura, Nakamura Hospital; S. Okamoto, Sanyo Osteoporosis Research Foundation Okamoto Naika Clinic; S. Nagai, Kagoshima Red Cross Hospital; H. Sakamoto, Sakamoto Medical Clinic. The present study was sponsored by ONO Pharmaceutical Co., Ltd. and Astellas Pharmaceutical.

Conflicts of interest None of the authors are or were employed by Astellas Pharmaceutical or Ono Pharmaceutical.

Drs. Matsumoto, Hagino, Shiraki, Fukunaga, Nakano, Takaoka, Ohashi and Nakamura have received consultant/honorarium fees from Astellas and Ono.

Open Access This article is distributed under the terms of the Creative Commons Attribution Noncommercial License which permits any noncommercial use, distribution, and reproduction in any medium, provided the original author(s) and source are credited.

\section{References}

1. Ettinger B, Black DM, Nevitt MC et al (1992) Contribution of vertebral deformities to chronic back pain and disability. The study of osteoporotic fractures research group. J Bone Miner Res 7:449-456
2. Ross PD, Fujiwara S, Huang C et al (1995) Vertebral fracture prevalence in women in Hiroshima compared to Caucasians or Japanese in the US. Int J Epidemiol 24:1171-1177

3. Lindsay R, Silverman SL, Cooper C et al (2001) Risk of new vertebral fracture in the year following a fracture. JAMA 285:320-323

4. Cauley JA, Thompson DE, Ensrud KC et al (2000) Risk of mortality following clinical fractures. Osteoporos Int 11:556-561

5. Ensrud KE, Thompson DE, Cauley JA et al (2000) Prevalent vertebral deformities predict mortality and hospitalization in older women with low bone mass. Fracture intervention trial research group. J Am Geriatr Soc 48:241-249

6. Yoshida Y, Moriya A, Kitamura K et al (1998) Responses of trabecular and cortical bone turnover and bone mass and strength to bisphosphonate YH529 in ovariohysterectomized beagles with calcium restriction. J Bone Miner Res 13:1011-1022

7. Dunford JE, Thompson K, Coxon FP et al (2001) Structureactivity relationships for inhibition of farnesyl diphosphate synthase in vitro and inhibition of bone resorption in vivo by nitrogen-containing bisphosphonates. J Pharmacol Exp Ther 296:235-242

8. Morii H, Nishizawa Y, Taketani Y et al (2002) A randomized controlled trial with ONO-5920 (Minodronate/YM529) in Japanese patients with postmenopausal osteoporosis. J Bone Miner Res 17(Suppl):S471

9. Orimo H, Hayashi Y, Fukunaga M et al (2001) Diagnostic criteria of primary osteoporosis: year 2000 revision. Osteoporosis diagnostic criteria review committee: Japanese society for bone mineral research. J Bone Miner Metab 19:331-937

10. Orimo H, Sugioka Y, Fukunaga M et al (1996) Diagnostic criteria of primary osteoporosis (1996 version). Osteoporosis diagnostic criteria review committee: Japanese society for bone and mineral research. Osteoporosis Jpn 4:643-653

11. Harris ST, Watts NB, Genant HK et al (1999) Effects of risedronate treatment on vertebral and nonvertebral fractures in women with postmenopausal osteoporosis. A randomized controlled trial. JAMA 282:1344-1352

12. Genant HK, Wu CY, Van Kuijk C et al (1993) Vertebral fracture assessment using a semiquantitative technique. J Bone Miner Res 8:1137-1148

13. Wu CY, Li J, Jergas M et al (1995) Comparison of semiquantitative and quantitative techniques for the assessment of prevalent and incident vertebral fractures. Osteoporos Int 5:354-370

14. James IT, Perrett D, Thompson PW (1990) Rapid assay for hard tissue collagen cross-links using isocratic ion-pair reversed-phase liquid chromatography. J Chromatogr 525:43-57

15. Chesnut CH, Skag A, Christiansen C et al (2004) Effects of oral ibandronate administered daily or intermittently on fracture risk in postmenopausal osteoporosis. J Bone Miner Res 19:1241-1249

16. Black DM, Delmas PD, Eastell R et al (2007) Once-yearly zoledronic acid for treatment of postmenopausal osteoporosis. $\mathrm{N}$ Engl J Med 356:1809-1822 